\title{
Existence results for nonlinear fractional boundary value problem involving generalized proportional derivative
}

\author{
Wafa Shammakh¹ and Hadeel Z. Alzumi ${ }^{1 *}$
}

"Correspondence:

hzalzumi@uj.edu.sa

${ }^{1}$ Mathematics Department, Faculty

of Science, University of Jeddah,

Jeddah, Saudi Arabia

\begin{abstract}
We introduce nonlinear fractional BVPs including a generalized proportional derivatives with nonlocal multipoint and substrip boundary conditions. The nonlinearities are defined on the Orlicz space and depend on the unknown function and its generalized derivative. Existence results for a nonlinear boundary value problem involving a proportional fractional derivative by utilizing some fixed point theorems are presented. The obtained results are new and are well illustrated with an example.
\end{abstract}

Keywords: Generalized proportional derivative; Fractional boundary value problem; Orlicz spaces

\section{Introduction}

The theory of fractional derivative first appeared in the 1690s by the correspondence between L'Hospital and Leibniz. After that, many researchers developed this area in different directions because of its wide application in solving practical problems in the fields of viscoelasticity, biological science, ecology, aerodynamics, etc. The recent history of fractional calculus can be found in [1]. During this development, a variety of initial and boundary conditions (BCs), such as classical, nonlocal, multipoint, periodic/anti-periodic and integral boundary conditions, were investigated. Many new results were obtained recently in fractional differential equations with nonlocal multipoint and with nonlocal multi-strip integral boundary conditions involving Caputo derivative; for example, see [2-6] and the references cited therein. In 2015, Caputo and Fabrizio [7] proposed a new definition of fractional derivative with a smooth kernel involving the exponential function. Other definition was introduced by Atangana and Baleanu [8] where the kernel appeared via the Mittag-Leffler function. These generalized fractional derivatives have been studied by many researchers. Recently, Jarad et al. [9] generated Caputo and Riemann-Liouville generalized proportional fractional (GPF) derivatives involving exponential functions in their kernels, thus the newly defined derivatives possess a semi-group property and they provide a generalization to the Caputo and Riemann-Liouville fractional derivatives and integrals. A variety of results can be found in the recent literature; for example, see [10-18] and the references therein.

(c) The Author(s) 2019. This article is distributed under the terms of the Creative Commons Attribution 4.0 International License (http://creativecommons.org/licenses/by/4.0/), which permits unrestricted use, distribution, and reproduction in any medium, provided you give appropriate credit to the original author(s) and the source, provide a link to the Creative Commons license, and indicate if changes were made. 
In this paper, we study the following fractional problem:

$$
\left\{\begin{array}{c}
{ }^{C} D^{q, \rho} x(t)=f\left(t, x(t), D^{p, \rho} x(t)\right), \quad 1<q \leq 2, t \in[0,1], p \in(0,1), \\
x(0)=\alpha_{1}, \\
{ }^{C} D^{r, \rho} x(1)=\alpha_{2} \int_{\zeta}^{\eta}{ }^{C} D^{r, \rho} x(s) d s+\alpha_{3} \sum_{i=1}^{m-2} \beta_{i}^{C} D^{r, \rho} x\left(\gamma_{i}\right), \\
0<\zeta<\eta<\gamma_{1}<\gamma_{2}<\cdots<\gamma_{m-2}<1, r \in(0,1)
\end{array}\right.
$$

where $\alpha_{i}(i=1,2,3)$ are positive real constants, $f$ is defined on an Orlicz space $L_{F}([0,1])$ and ${ }^{C} D^{q, \rho}$ denotes the generalized proportional fractional derivative of Caputo type. It is imperative to mention that the nonlocal multipoint and substrip BC (1) can be explained in the sense that the linear combinations of values of the GPFD of Caputo type of the unknown function at the right end point $t=1$ of the interval under consideration is proportional to the sum of the values of the GPFD of the unknown function on the strip $(\zeta, \eta)$ and scalar multiplies of discrete values of the unknown function at $\gamma_{i}(i=1,2, \ldots, m-2)$.

This kind of $\mathrm{BC}$ plays a key role in formulating chemical, physical, or other processes involving some peculiarities occurring inside the domain. On the other hand, distinct applications of applied sciences such as population dynamics, chemical engineering, blood flow problems, can be represented by an integral BC. For more details, for example, see $[19,20]$.

In another direction, in 1931, Birnbaum and Orlicz [21] introduced a generalization of the classical Lebesgue spaces $L_{p}, 1<p<+\infty$. This generalization is called an Orlicz space and is found by replacing the function $x^{p}$ in the definition of $L_{p}$ by a more general convex function $F$, which is called the $N$-function. Recently, the existence of solutions of differential equations was investigated; see, for example, [22, 23].

In the present paper, we study Caputo type fractional differential equations with nonlocal multipoint and substrips boundary conditions (1) involving the generalized proportional derivative and let $f$ be a function in an Orlicz space. We discuss the existence of a solution for a nonlinear boundary value problem using some fixed point theorems. Finally, we present an example for illustration of the main result.

\section{Preliminaries}

We recall some basic concepts needed throughout this paper including Orlicz spaces and fractional calculus. For more details as regards Orlicz space, the reader can refer to [24] and for fractional calculus one can see [9, 25-27].

Definition 1 Let $\varphi:[0, \infty) \rightarrow[0, \infty)$ be right continuous, monotone, increasing function with

(i) $\varphi(0)=0$,

(ii) $\lim _{t \rightarrow \infty} \varphi(t)=\infty$,

(iii) $\varphi(t)>0$ whenever $t>0$.

Then the function defined by

$$
F(x)=\int_{0}^{x} \varphi(t) d t, \quad x \geq 0,
$$

is called the $N$-function. Alternatively, the function $F$ is an $N$-function iff $F$ is continuous, even, and convex with 
(i) $\lim _{x \rightarrow 0} \frac{F(x)}{x}=0$,

(ii) $\lim _{x \rightarrow \infty} \frac{F(x)}{x}=\infty$,

(iii) $F(x)>0$ if $x>0$.

Definition 2 For an $N$-function, we define

$$
F^{*}(x)=\int_{0}^{x} \varphi^{-1}(t) d t, \quad x \geq 0,
$$

where $\varphi^{-1}$ is the right inverse of the right derivative of $F$, is called the complementary of $F$ and it satisfies the condition

$$
F^{*}(x)=\sup \{t x-F(t): t \geq 0\}, \quad \forall x \geq 0 .
$$

(i) The function $F^{*}$ is also $N$-function.

(ii) The complementary pairs $F$ and $F^{*}$ satisfy the following Young inequality:

$$
x t \leq F(x)+F^{*}(t), \quad \forall x, t \geq 0 .
$$

Definition 3 A function $F:[0, \infty) \rightarrow[0, \infty)$ is called a Young function if it is convex and satisfies the conditions

$$
F(0)=\lim _{x \rightarrow 0^{+}} F(x)=0 \text { and } \quad \lim _{x \rightarrow \infty} F(x)=\infty .
$$

Remark 4 If a Young function $F$ satisfies $\varphi(0)=0 \Longleftrightarrow x=0$, then the conditions $\lim _{x \rightarrow 0} \frac{F(x)}{x}=0$ and $\lim _{x \rightarrow \infty} \frac{F(x)}{x}=\infty$ hold; then $F$ is called an $N$-function.

Definition 5 Let $F$ be an $N$-function and let $F^{*}$ be its complement. Then $F$ is said to satisfy the $\triangle_{2}$-condition if

$$
\limsup _{x \rightarrow \rightarrow \infty} \frac{F(2 x)}{F(x)}<\infty
$$

that is, there is a $k>0$ such that $F(2 x) \leq k F(x)$ for large values of $x$.

Definition 6 (Orlicz space) For an $N$-function $F$, the Orlicz space $L_{F}([0,1])$ is the space of measurable functions $u:[0,1] \rightarrow \mathbb{R}$ such that $\int_{0}^{1} F(|u(x)|) d x<\infty$. This space endowed with the Luxemburg norm, i.e.,

$$
\|u\|_{F}=\inf \left\{\lambda>0: \int_{0}^{1} F\left(\frac{|u(x)|}{\lambda}\right) d x \leq 1\right\}
$$

and the pair $\left(L_{F}([0,1]),\|u\|_{F}\right)$ is a Banach space.

For an Orlicz space, the Hölder inequality holds, that is,

$$
\int_{0}^{1} u v d x \leq 2\|u\|_{F}\|v\|_{F^{*}},
$$

where $u \in L_{F}([0,1])$ and $v \in L_{F^{*}}([0,1])$. 


\section{Definition 7}

1. For an at least $n$-times continuously differentiable function $u:[0, \infty) \rightarrow \mathbb{R}$, the Caputo derivative of fractional order $q$ is defined by

$$
{ }^{c} D^{q} u(x)=\frac{1}{\Gamma(n-q)} \int_{0}^{x}(x-t)^{n-1-q} u^{(n)}(t) d t, \quad n-1<q<n, n=[q]+1, q>0,
$$

where $[q]$ denotes the integer part of the real number $q$ and $\Gamma$ denotes the gamma function.

2. The Riemann-Liouville fractional integral of order $q$ for the continuous function $u$ is defined by

$$
I^{q} u(x)=\frac{1}{\Gamma(q)} \int_{0}^{x}(x-t)^{q-1} u(t) d t, \quad q>0
$$

provided the right-hand side is pointwise defined on $(0, \infty)$.

Definition 8 (The GPF integral [9]) For $\rho \in(0,1]$ and $\alpha \in \mathbb{C}$ with $\Re(\alpha)>0$, we define the left generalized proportional fractional integral of $f$ starting by $a$,

$$
\left({ }_{a} I^{\alpha, \rho} f\right)(x)=\frac{1}{\rho^{\alpha} \Gamma(\alpha)} \int_{a}^{x} e^{\frac{\rho-1}{\rho}(x-t)}(x-t)^{\alpha-1} f(t) d t .
$$

Definition 9 (The GPF dervative of Caputo type) For $\rho \in(0,1]$ and $\alpha \in \mathbb{C}$ with $\Re(\alpha)>0$, we define the left generalized proportional fractional derivative of Capotu type starting by $a$,

$$
\begin{aligned}
\left({ }_{a}^{C} D^{\alpha, \rho} f\right)(x) & ={ }_{a} I^{n-\alpha, \rho}\left(D^{n, \rho} f\right)(x) \\
& =\frac{1}{\rho^{n-\alpha} \Gamma(n-\alpha)} \int_{a}^{x} e^{\frac{\rho-1}{\rho}(x-t)}(x-t)^{n-\alpha-1}\left(D^{n, \rho} f\right)(t) d t,
\end{aligned}
$$

where $n=[\Re(\alpha)]+1$.

Theorem 10 ([9]) For $\rho \in(0,1]$ and $n=[\Re(\alpha)]+1$, we have

$$
{ }_{a} I^{n-\alpha, \rho}\left(D^{n, \rho} f\right)(x)=f(x)-\sum_{k=0}^{n-1} \frac{\left(D^{k, \rho} f\right)(t)}{\rho^{k} k !}(x-t)^{k} e^{\frac{\rho-1}{\rho}(x-t)}
$$

We base our considerations on the following fixed point theorems in our main results.

Theorem 11 (Krasnoselskii's fixed point theorem [28]) Let $\mathcal{P}$ be a closed, convex, bounded and nonempty subset of a Banach space X. Let $T_{1}, T_{2}$ be operators such that

(i) $T_{1}\left(u_{1}\right)+T_{2}\left(u_{2}\right)$ belong to $P$ whenever $u_{1}, u_{2} \in P$.

(ii) $T_{1}$ is a compact and continuous and $T_{2}$ is a contraction mapping.

Then there exists $u_{0} \in P$ such that $u_{0}=T_{1}\left(u_{0}\right)+T_{2}\left(u_{0}\right)$.

Theorem 12 (Schaefer's fixed point theorem [28]) Let $X$ be a Banach space. Assume that $T: X \rightarrow X$ is a completely continuous operator and the set $V=\{u \in X: u=\varepsilon T u, 0<\varepsilon<1\}$ is bounded. Then $T$ has a fixed point in $X$. 
For convenience, we denote

$$
\begin{aligned}
A_{1}= & \frac{(1-\rho) e^{\frac{\rho-1}{\rho}}}{\rho^{1-r} \Gamma(2-r)}-\frac{\alpha_{2}(1-\rho)}{\rho^{1-r} \Gamma(2-r)} \int_{\zeta}^{\eta} e^{\frac{\rho-1}{\rho} s} s^{2-r} d s \\
& -\alpha_{3} \sum_{i=1}^{m-2} \beta_{i} \frac{(1-\rho) e^{\frac{\rho-1}{\rho} \gamma_{i}}}{\rho^{1-r} \Gamma(2-r)}\left(\gamma_{i}\right)^{2-r}, \\
A_{2}= & \frac{\alpha_{1} \alpha_{2}(1-\rho)}{\rho^{1-r} \Gamma(3-r)}\left[e^{\frac{\rho-1}{\rho} \eta} \eta^{2-r}-e^{\frac{\rho-1}{\rho} \zeta} \zeta^{2-r}\right]+\frac{\alpha_{1} \alpha_{2}(1-\rho)^{2}}{\rho^{2-r} \Gamma(3-r)} \\
& +\alpha_{3} \sum_{i=1}^{m-2} \beta_{i} \frac{\alpha_{1}(1-\rho)}{\rho^{1-r} \Gamma(2-r)} e^{\frac{\rho-1}{\rho} \gamma_{i}}\left(\gamma_{i}\right)^{1-r}-\frac{\alpha_{1}(1-\rho) e^{\frac{\rho-1}{\rho}}}{\rho^{1-r} \Gamma(2-r)}, \\
A^{*}= & \frac{A_{2}}{A_{1}} .
\end{aligned}
$$

Lemma 13 For any $f \in L_{F}([0,1])$, the solution of the fractional boundary problem

$$
\left\{\begin{array}{l}
{ }^{C} D^{q, \rho} x(t)=f(t), \quad 1<q \leq 2, t \in[0,1], \\
x(0)=\alpha_{1}, \\
{ }^{C} D^{r, \rho} x(1)=\alpha_{2} \int_{\zeta}^{\eta}{ }^{C} D^{r, \rho} x(s) d s+\alpha_{3} \sum_{i=1}^{m-2} \beta_{i}{ }^{C} D^{r, \rho} x\left(\gamma_{i}\right), \\
0<\zeta<\eta<\gamma_{1}<\gamma_{2}<\cdots<\gamma_{m-2}<1, \quad r \in(0,1),
\end{array}\right.
$$

is

$$
\begin{aligned}
x(t)= & \frac{1}{\rho^{q} \Gamma(q)} \int_{0}^{t} e^{\frac{\rho-1}{\rho}(t-s)}(t-s)^{q-1} f\left(s, x(s),{ }^{C} D^{p, \rho} x(s)\right) d s \\
& +\mu_{1}(t)\left[\alpha_{2} \int_{\zeta}^{\eta}\left(\int_{0}^{s} \frac{e^{\frac{\rho-1}{\rho}(s-u)}(s-u)^{q-r-1}}{\rho^{q-r} \Gamma(q-r)} f\left(u, x(u),{ }^{C} D^{p, \rho} x(u)\right) d u\right) d s\right. \\
& +\alpha_{3} \sum_{i=1}^{m-2} \beta_{i} \int_{0}^{\gamma_{i}} \frac{e^{\frac{\rho-1}{\rho}\left(\gamma_{i}-s\right)}\left(\gamma_{i}-s\right)^{q-r-1}}{\rho^{q-r} \Gamma(q-r)} f\left(s, x(s),{ }^{C} D^{p, \rho} x(s)\right) d s \\
& \left.-\int_{0}^{1} \frac{e^{\frac{\rho-1}{\rho}(1-s)}(1-s)^{q-r-1}}{\rho^{q-r} \Gamma(q-r)} f\left(s, x(s),{ }^{C} D^{p, \rho} x(s)\right) d s\right]+\mu_{2}(t),
\end{aligned}
$$

where

$$
\begin{aligned}
& \mu_{1}(t)=\frac{1}{A_{1}} t e^{\frac{\rho-1}{\rho} t}, \quad A_{1} \neq 0, \\
& \mu_{2}(t)=\left(\alpha_{1}+A^{*} t\right) e^{\frac{\rho-1}{\rho} t}
\end{aligned}
$$

Proof The general solution of the fractional differential equation (2) is given by

$$
x(t)=\left(I^{q, \rho} y\right)(t)+\sum_{k=0}^{1} c_{k} t^{k} e^{\frac{\rho-1}{\rho} t},
$$


that is,

$$
x(t)=\left(I^{q, \rho} y\right)(t)+\left(c_{0}+c_{1} t\right) e^{\frac{\rho-1}{\rho} t},
$$

where $c_{0}, c_{1} \in \mathbb{R}$ are arbitrary constants. Using the condition $x(0)=\alpha_{1}$, we get $c_{0}=\alpha_{1}$. Now, by applying the second boundary condition, we have

$$
\begin{aligned}
c_{1}= & A^{*}+\frac{\alpha_{2}}{A_{1}} \int_{\zeta}^{\eta}\left(I^{q-r, \rho} y\right)(s) d s+\frac{\alpha_{3}}{A_{1}} \sum_{i=1}^{m-2} \beta_{i}\left(I^{q-r, \rho} y\right)\left(\gamma_{i}\right) \\
& -\frac{1}{A_{1}}\left(I^{q-r, \rho} y\right)(1) .
\end{aligned}
$$

Substituting from $c_{0}$ and $c_{1}$ in (6), we get (3).

\section{Existence results}

In this section, we discuss the existence of solutions to the BVP (1). We shall assume that $f$ is in the Orlicz space $L_{F}[0,1]$. For $0<p<1$, let $X=\left\{x: x,{ }^{C} D^{p, \rho} x \in C([0,1], \mathbb{R})\right\}$ denotes the Banach space of all continuous functions on $[0,1]$ into $\mathbb{R}$ endowed with the norm $\|x\|=\sup \left\{|x(t)|+\left|{ }^{C} D^{p, \rho} x(t)\right|, t \in[0,1]\right\}$.

Now, we define an operator $T: X \rightarrow X$ associated with the problem (1) by

$$
\begin{aligned}
(T x)(t)= & \frac{1}{\rho^{q} \Gamma(q)} \int_{0}^{t} e^{\frac{\rho-1}{\rho}(t-s)}(t-s)^{q-1} f\left(s, x(s),{ }^{C} D^{p, \rho} x(s)\right) d s \\
& +\mu_{1}(t)\left[\alpha_{2} \int_{\zeta}^{\eta}\left(\int_{0}^{s} \frac{e^{\frac{\rho-1}{\rho}(s-u)}(s-u)^{q-r-1}}{\rho^{q-r} \Gamma(q-r)} f\left(u, x(u),{ }^{C} D^{p, \rho} x(u)\right) d u\right) d s\right. \\
& +\alpha_{3} \sum_{i=1}^{m-2} \beta_{i} \int_{0}^{\gamma_{i}} \frac{e^{\frac{\rho-1}{\rho}\left(\gamma_{i}-s\right)}\left(\gamma_{i}-s\right)^{q-r-1}}{\rho^{q-r} \Gamma(q-r)} f\left(s, x(s),{ }^{C} D^{p, \rho} x(s)\right) d s \\
& \left.-\int_{0}^{1} \frac{e^{\frac{\rho-1}{\rho}(1-s)}(1-s)^{q-r-1}}{\rho^{q-r} \Gamma(q-r)} f\left(s, x(s),{ }^{C} D^{p, \rho} x(s)\right) d s\right]+\mu_{2}(t),
\end{aligned}
$$

where $\mu_{1}, \mu_{2}$ are given by (4) and (5). Therefore, the problem (1) has solutions if and only if the operator $T$ has a fixed point.

Lemma 14 Let $q \in(1,2]$ and $r \in(0,1)$. Let $F$ be a Young function which has a Young complement $F^{*}$ satisfying

$$
\int_{0}^{t} F^{*}\left(s^{q-1}\right) d s<\infty, \quad \text { and } \quad \int_{0}^{t} F^{*}\left(s^{q-r-1}\right) d s<\infty, \quad t>0 .
$$

Then the operator $T$ exists and is well defined.

Proof Let $q \in(1,2], r \in(0,1)$ and $x \in X$. Define a function

$$
\psi_{1}(s)= \begin{cases}s^{q-1} & \text { if } s \in[0, t], t>0 \\ 0 & \text { otherwise }\end{cases}
$$


We show that $\psi_{1} \in L_{F^{*}}[0,1]$. By using appropriate substitution and properties of the Young functions, one obtains

$$
\begin{aligned}
\int_{0}^{1} F^{*}\left(\frac{\left|\psi_{1}(s)\right|}{\alpha}\right) d s & =\int_{0}^{t} F^{*}\left(\frac{(t-s)^{q-1}}{\alpha}\right) d s \\
& =\left(\frac{1}{\alpha}\right)^{\frac{1}{q-1}} \int_{0}^{\alpha^{\frac{1}{q-1}} t} F^{*}\left(s^{q-1}\right) d s ;
\end{aligned}
$$

by the assumption of the theorem, we get $\psi_{1} \in L_{F^{*}}[0,1]$. Similarly, set

$$
\psi_{2}(s)= \begin{cases}s^{q-r-1} & \text { if } s \in[0, t], t>0 \\ 0 & \text { otherwise }\end{cases}
$$

one can get $\psi_{2} \in L_{F^{*}}[0,1]$. Next, we show that $T$ is well defined, i.e., $T x(t) \in C([0,1], \mathbb{R})$. Let $0 \leq \tau<t \leq 1$. Then

$$
\begin{aligned}
& |(T x)(t)-(T x)(\iota)| \\
& \leq \mid \frac{1}{\rho^{q} \Gamma(q)} \int_{0}^{\iota} e^{\frac{\rho-1}{\rho}(t-s)}(t-s)^{q-1} f\left(s, x(s), D^{p, \rho} x(s)\right) d s \\
& -\frac{1}{\rho^{q} \Gamma(q)} \int_{0}^{\tau} e^{\frac{\rho-1}{\rho}(\iota-s)}(\tau-s)^{q-1} f\left(s, x(s), D^{p, \rho} x(s)\right) d s \mid \\
& +\left|\mu_{1}(t)-\mu_{1}(\iota)\right|\left[\alpha_{2} \int_{\zeta}^{\eta}\left(\int_{0}^{s} \frac{\left|e^{\frac{\rho-1}{\rho}(s-u)}\right|(s-u)^{q-r-1}}{\rho^{q-r} \Gamma(q-r)}\left|f\left(u, x(u), D^{p, \rho} x(u)\right)\right| d u\right) d s\right. \\
& +\alpha_{3} \sum_{i=1}^{m-2} \beta_{i} \int_{0}^{\gamma_{i}} \frac{\left|e^{\frac{\rho-1}{\rho}\left(\gamma_{i}-s\right)}\right|\left(\gamma_{i}-s\right)^{q-r-1}}{\rho^{q-r} \Gamma(q-r)}\left|f\left(s, x(s), D^{p, \rho} x(s)\right)\right| d s \\
& \left.-\int_{0}^{1} \frac{\left|e^{\frac{\rho-1}{\rho}(1-s)}\right|(1-s)^{q-r-1}}{\rho^{q-r} \Gamma(q-r)}\left|f\left(s, x(s), D^{p, \rho} x(s)\right)\right| d s\right]+\left|\mu_{2}(t)-\mu_{2}(\iota)\right| \\
& =\frac{1}{\rho^{q} \Gamma(q)} \int_{0}^{t}\left|e^{\frac{\rho-1}{\rho}(t-s)}\right|(t-s)^{q-1}\left|f\left(s, x(s), D^{p, \rho} x(s)\right)\right| d s \\
& +\frac{1}{\rho^{q} \Gamma(q)} \int_{\tau}^{t}\left|e^{\frac{\rho-1}{\rho}(t-s)}\right|(t-s)^{q-1}\left|f\left(s, x(s), D^{p, \rho} x(s)\right)\right| d s \\
& -\frac{1}{\rho^{q} \Gamma(q)} \int_{0}^{\tau}\left|e^{\frac{\rho-1}{\rho}(\tau-s)}\right|(\tau-s)^{q-1}\left|f\left(s, x(s), D^{p, \rho} x(s)\right)\right| d s \\
& +\left|\mu_{1}(t)-\mu_{1}(\tau)\right|\left[\alpha_{2} \int_{\zeta}^{\eta}\left(\int_{0}^{s} \frac{\left|e^{\frac{\rho-1}{\rho}(s-u)}\right|(s-u)^{q-r-1}}{\rho^{q-r} \Gamma(q-r)}\left|f\left(u, x(u), D^{p, \rho} x(u)\right)\right| d u\right) d s\right. \\
& +\alpha_{3} \sum_{i=1}^{m-2} \beta_{i} \int_{0}^{\gamma_{i}} \frac{\left|e^{\frac{\rho-1}{\rho}\left(\gamma_{i}-s\right)}\right|\left(\gamma_{i}-s\right)^{q-r-1}}{\rho^{q-r} \Gamma(q-r)}\left|f\left(s, x(s), D^{p, \rho} x(s)\right)\right| d s \\
& \left.-\int_{0}^{1} \frac{\left|e^{\frac{\rho-1}{\rho}(1-s)}\right|(1-s)^{q-r-1}}{\rho^{q-r} \Gamma(q-r)}\left|f\left(s, x(s), D^{p, \rho} x(s)\right)\right| d s\right]+\left|\mu_{2}(t)-\mu_{2}(\tau)\right| .
\end{aligned}
$$


Since $\left|e^{\frac{\rho-1}{\rho} t}\right| \leq 1$, we have

$$
\begin{aligned}
\mid(T x)( & t)-(T x)(\iota) \mid \\
\leq & \frac{1}{\rho^{q} \Gamma(q)} \int_{0}^{\tau}\left|(t-s)^{q-1}-(\tau-s)^{q-1}\right|\left|f\left(s, x(s), D^{p, \rho} x(s)\right)\right| d s \\
& +\frac{1}{\rho^{q} \Gamma(q)} \int_{\tau}^{t}\left|(t-s)^{q-1}\right|\left|f\left(s, x(s), D^{p, \rho} x(s)\right)\right| d s \\
& +\left|\mu_{1}(t)-\mu_{1}(\tau)\right| \\
& \times\left[\frac{\alpha_{2}}{\rho^{q-r} \Gamma(q-r)} \int_{\zeta}^{\eta}\left(\int_{0}^{s}\left|(s-u)^{q-r-1}\right|\left|f\left(u, x(u), D^{p, \rho} x(u)\right)\right| d u\right) d s\right. \\
& +\frac{\alpha_{3}}{\rho^{q-r} \Gamma(q-r)} \sum_{i=1}^{m-2} \beta_{i} \int_{0}^{\gamma_{i}}\left|\left(\gamma_{i}-s\right)^{q-r-1}\right|\left|f\left(s, x(s), D^{p, \rho} x(s)\right)\right| d s \\
& \left.-\frac{1}{\rho^{q-r} \Gamma(q-r)} \int_{0}^{1}\left|(1-s)^{q-r-1}\right|\left|f\left(s, x(s), D^{p, \rho} x(s)\right)\right| d s\right]+\left|\mu_{2}(t)-\mu_{2}(\tau)\right| \\
& +\frac{1}{\rho^{q-r} \Gamma(q-r)} \sum_{i=1}^{m-2} \beta_{i} \int_{0}^{\gamma_{i}}\left|\left(\gamma_{i}-s\right)^{q-r-1}\right|\left|f\left(s, x(s), D^{p, \rho} x(s)\right)\right| d s \\
& +\frac{\alpha_{2}}{\rho^{q} \Gamma(q)} \int_{0}\left[\chi_{1}(s)+\chi_{2}(s)\right]\left|f\left(s, x(s), D^{p, \rho} x(s)\right)\right| d s \\
& +\left|\mu_{1}(t)-\mu_{1}(\tau)\right| \\
& \times\left[\frac{1}{\rho^{q-r} \Gamma(q-r)} \int_{\zeta}^{\eta}\left(\int_{0}^{s}\left|(s-u)^{q-r-1}\right|\left|f\left(u, x(u), D^{p, \rho} x(u)\right)\right| d u\right) d s\right. \\
& {\left[(1-s)^{q-r-1}|| f\left(s, x(s), D^{p, \rho} x(s)\right) \mid d s\right]+\left|\mu_{2}(t)-\mu_{2}(\tau)\right|, }
\end{aligned}
$$

where

$$
\chi_{1}(s)= \begin{cases}\left|(t-s)^{q-1}-(\tau-s)^{q-1}\right| & \text { if } s \in[0, \tau] \\ 0 & \text { otherwise }\end{cases}
$$

and

$$
\chi_{2}(s)= \begin{cases}\left|(t-s)^{q-1}\right| & \text { if } s \in[\tau, t] \\ 0 & \text { otherwise }\end{cases}
$$

The functions $\chi_{i}, i=1,2$ belong to $L_{F^{*}}[0,1]$ with $\left\|\chi_{i}\right\|_{F^{*}} \leq h(|t-\tau|), i=1,2$ where $h: \mathbb{R}^{+} \rightarrow$ $\mathbb{R}^{+}$is a continuous, increasing, function with $h(0)=0$. Using the Hölder inequality, we have

$$
\begin{aligned}
& |(T x)(t)-(T x)(\tau)| \\
& \quad \leq \frac{2}{\rho^{q} \Gamma(q)}\left[\left\|\chi_{1}\right\|_{F^{*}}+\left\|\chi_{2}\right\|_{F^{*}}\right]\|f\|_{F}
\end{aligned}
$$




$$
\begin{aligned}
& +|t-\tau|\left[\left(\frac { 1 } { | A _ { 1 } | } \left[\frac{\alpha_{2}}{\rho^{q-r} \Gamma(q-r+2)}\left(\eta^{q-r+1}-\zeta^{q-r+1}\right)\right.\right.\right. \\
& \left.\left.\left.+\frac{\alpha_{3}}{\rho^{q-r} \Gamma(q-r+1)} \sum_{i=1}^{m-2} \beta_{i} \gamma_{i}^{q-r}-\frac{1}{\rho^{q-r} \Gamma(q-r+1)}\right]\right)\|f\|_{F}+\left|A^{*}\right|\right]
\end{aligned}
$$

then, for $0<|t-\tau|<\delta$ and by the continuity of $h$, we see that $T x$ is continuous, which completes the proof.

Our first existence result is based on Schaefer's fixed point theorem.

Theorem 15 Assume that there exists $\lambda \in C\left([0,1], \mathbb{R}^{+}\right)$such that

$$
\left|f\left(t, x(t),{ }^{C} D^{p, \rho} x(t)\right)\right| \leq \lambda(t) \quad \text { for } t \in[0,1] \text { with }\|\lambda\|=\max _{t \in[0,1]}|\lambda(t)| .
$$

Then the problem (1) has at least one solution on $[0,1]$.

Proof We shall show that the operator $T$ is completely continuous. Let $G \subset X$ be a bounded set. Then, for all $x \in G$, we get

$$
\begin{aligned}
|(T x)(t)| \leq & \frac{1}{\rho^{q} \Gamma(q)} \int_{0}^{t}\left|e^{\frac{\rho-1}{\rho}(t-s)}\right|(t-s)^{q-1}\left|f\left(s, x(s), D^{p, \rho} x(s)\right)\right| d s \\
& +\left|\mu_{1}(t)\right|\left[\alpha_{2} \int_{\zeta}^{\eta}\left(\int_{0}^{s} \frac{\left|e^{\frac{\rho-1}{\rho}(s-u)}\right|(s-u)^{q-r-1}}{\rho^{q-r} \Gamma(q-r)}\left|f\left(u, x(u), D^{p, \rho} x(u)\right)\right| d u\right) d s\right. \\
& +\alpha_{3} \sum_{i=1}^{m-2} \beta_{i} \int_{0}^{\gamma_{i}} \frac{\left|e^{\frac{\rho-1}{\rho}\left(\gamma_{i}-s\right)}\right|\left(\gamma_{i}-s\right)^{q-r-1}}{\rho^{q-r} \Gamma(q-r)}\left|f\left(s, x(s), D^{p, \rho} x(s)\right)\right| d s \\
& \left.-\int_{0}^{1} \frac{\left|e^{\frac{\rho-1}{\rho}(1-s)}\right|(1-s)^{q-r-1}}{\rho^{q-r} \Gamma(q-r)}\left|f\left(s, x(s), D^{p, \rho} x(s)\right)\right| d s\right] \\
& +\left|\mu_{2}(t)\right| ;
\end{aligned}
$$

by using the condition $\left|f\left(t, x(t),{ }^{C} D^{p, \rho} x(t)\right)\right| \leq \lambda(t)$ for $t \in[0,1]$, we obtain

$$
\begin{aligned}
|(T x)(t)| \leq & \frac{1}{\rho^{q} \Gamma(q)} \int_{0}^{t}\left|e^{\frac{\rho-1}{\rho}(t-s)}\right|(t-s)^{q-1}|\lambda(s)| d s \\
& +\left|\mu_{1}(t)\right|\left[\alpha_{2} \int_{\zeta}^{\eta}\left(\int_{0}^{s} \frac{\left|e^{\frac{\rho-1}{\rho}(s-u)}\right|(s-u)^{q-r-1}}{\rho^{q-r} \Gamma(q-r)}|\lambda(u)| d u\right) d s\right. \\
& +\alpha_{3} \sum_{i=1}^{m-2} \beta_{i} \int_{0}^{\gamma_{i}} \frac{\left|e^{\frac{\rho-1}{\rho}\left(\gamma_{i}-s\right)}\right|\left(\gamma_{i}-s\right)^{q-r-1}}{\rho^{q-r} \Gamma(q-r)}|\lambda(s)| d s \\
& \left.-\int_{0}^{1} \frac{\left|e^{\frac{\rho-1}{\rho}(1-s)}\right|(1-s)^{q-r-1}}{\rho^{q-r} \Gamma(q-r)}|\lambda(s)| d s\right]+\left|\mu_{2}(t)\right| .
\end{aligned}
$$


From the above inequality, we obtain

$$
\begin{aligned}
& \|T x\| \leq \frac{\|\lambda\|}{\rho^{q} \Gamma(q)} \int_{0}^{t}\left|e^{\frac{\rho-1}{\rho}(t-s)}\right|(t-s)^{q-1} d s \\
& +\sup _{t \in[0,1]}\left|\mu_{1}(t)\right|\left[\frac{\alpha_{2}\|\lambda\|}{\rho^{q-r} \Gamma(q-r)} \int_{\zeta}^{\eta}\left(\int_{0}^{s}\left|e^{\frac{\rho-1}{\rho}(s-u)}\right|(s-u)^{q-r-1} d u\right) d s\right. \\
& +\frac{\alpha_{3}\|\lambda\|}{\rho^{q-r} \Gamma(q-r)} \sum_{i=1}^{m-2} \beta_{i} \int_{0}^{\gamma_{i}}\left|e^{\frac{\rho-1}{\rho}\left(\gamma_{i}-s\right)}\right|\left(\gamma_{i}-s\right)^{q-r-1} d s \\
& \left.-\frac{\|\lambda\|}{\rho^{q-r} \Gamma(q-r)} \int_{0}^{1}\left|e^{\frac{\rho-1}{\rho}(1-s)}\right|(1-s)^{q-r-1} d s\right]+\sup _{t \in[0,1]}\left|\mu_{2}(t)\right| \\
& \leq \frac{\|\lambda\|}{\rho^{q} \Gamma(q+1)} t^{q}+\bar{\mu}_{1}\left[\frac{\alpha_{2}\|\lambda\|}{\rho^{q-r} \Gamma(q-r+1)} \int_{\zeta}^{\eta} s^{q-r} d s\right. \\
& \left.+\frac{\alpha_{3}\|\lambda\|}{\rho^{q-r} \Gamma(q-r+1)} \sum_{i=1}^{m-2} \beta_{i} \gamma_{i}^{q-r}-\frac{\|\lambda\|}{\rho^{q-r} \Gamma(q-r+1)}\right]+\bar{\mu}_{2} \\
& \leq \frac{\|\lambda\|}{\rho^{q} \Gamma(q+1)}+\bar{\mu}_{1}\left[\frac{\alpha_{2}\|\lambda\|}{\rho^{q-r} \Gamma(q-r+2)}\left(\eta^{q-r+1}-\zeta^{q-r+1}\right)\right. \\
& \left.+\frac{\alpha_{3}\|\lambda\|}{\rho^{q-r} \Gamma(q-r+1)} \sum_{i=1}^{m-2} \beta_{i} \gamma_{i}^{q-r}-\frac{\|\lambda\|}{\rho^{q-r} \Gamma(q-r+1)}\right]+\bar{\mu}_{2} \\
& =M_{1} \text {. }
\end{aligned}
$$

Now,

$$
\begin{aligned}
\left({ }^{C} D^{p, \rho} T x\right)(t) & \left({ }^{C} D^{p, \rho}\left(I^{q, \rho} f\right)\right)(t) \\
& +{ }^{C} D^{p, \rho}\left(\mu _ { 1 } ( t ) \left[\alpha_{2} \int_{\zeta}^{\eta}\left(\int_{0}^{s} \frac{e^{\frac{\rho-1}{\rho}(s-u)}(s-u)^{q-r-1}}{\rho^{q-r} \Gamma(q-r)} f\left(u, x(u), D^{p, \rho} x(u)\right) d u\right) d s\right.\right. \\
& +\alpha_{3} \sum_{i=1}^{m-2} \beta_{i} \int_{0}^{\gamma_{i}} \frac{e^{\frac{\rho-1}{\rho}\left(\gamma_{i}-s\right)}\left(\gamma_{i}-s\right)^{q-r-1}}{\rho^{q-r} \Gamma(q-r)} f\left(s, x(s), D^{p, \rho} x(s)\right) d s \\
& \left.\left.-\int_{0}^{1} \frac{e^{\frac{\rho-1}{\rho}(1-s)}(1-s)^{q-r-1}}{\rho^{q-r} \Gamma(q-r)} f\left(s, x(s), D^{p, \rho} x(s)\right) d s\right]\right)+{ }^{C} D^{p, \rho} \mu_{2}(t) \\
& \frac{1}{\rho^{1-p}} \Gamma(1-p) \int_{0}^{t} e^{\frac{\rho-1}{\rho}(t-s)}(t-s)^{-p} D^{\rho}\left(I^{q, \rho} f\right)(s) d s \\
& +{ }^{C} D^{p, \rho} \mu_{1}(t)\left[\alpha_{2} \int_{\zeta}^{\eta}\left(\int_{0}^{s} \frac{e^{\frac{\rho-1}{\rho}(s-u)}(s-u)^{q-r-1}}{\rho^{q-r} \Gamma(q-r)} f\left(u, x(u), D^{p, \rho} x(u)\right) d u\right) d s\right. \\
& +\alpha_{3} \sum_{i=1}^{m-2} \beta_{i} \int_{0}^{\gamma_{i}} \frac{e^{\frac{\rho-1}{\rho}\left(\gamma_{i}-s\right)}\left(\gamma_{i}-s\right)^{q-r-1}}{\rho^{q-r} \Gamma(q-r)} f\left(s, x(s), D^{p, \rho} x(s)\right) d s \\
& \left.-\int_{0}^{1} \frac{e^{\frac{\rho-1}{\rho}(1-s)}(1-s)^{q-r-1}}{\rho^{q-r} \Gamma(q-r)} f\left(s, x(s), D^{p, \rho} x(s)\right) d s\right]+{ }^{C} D^{p, \rho} \mu_{2}(t) .
\end{aligned}
$$


Since $D^{p}\left(I^{q, \rho} f\right)(s)=\frac{\|\lambda\|}{\rho^{q-1} \Gamma(q)} s^{q-1},{ }^{C} D^{p, \rho} \mu_{1}(t)=\frac{t^{1-p}}{A_{1} \rho^{-p} \Gamma(2-p)}$ and ${ }^{C} D^{p, \rho} \mu_{2}(t)=\frac{A^{*} t^{1-p} e^{\frac{\rho-1}{\rho} t}}{\rho^{-p} \Gamma(2-p)}$, Eq. (10) becomes

$$
\begin{aligned}
\left|{ }^{C} D^{p, \rho} T x(t)\right| \leq & \frac{\|\lambda\| t^{q-1}}{\rho^{q-p} \Gamma(q) \Gamma(1-p)} \int_{0}^{t}\left|e^{\frac{\rho-1}{\rho}(t-s)}\right|(t-s)^{-p} d s \\
& +\frac{t^{1-p}}{A_{1} \rho^{-p} \Gamma(2-p)}\left[\frac{\alpha_{2}\|\lambda\|}{\rho^{q-r} \Gamma(q-r+2)}\left(\eta^{q-r+1}-\zeta^{q-r+1}\right)\right. \\
& \left.+\frac{\alpha_{3}\|\lambda\|}{\rho^{q-r} \Gamma(q-r+1)} \sum_{i=1}^{m-2} \beta_{i} \gamma_{i}^{q-r}-\frac{\|\lambda\|}{\rho^{q-r} \Gamma(q-r+1)}\right] \\
& +\frac{A^{*} t^{1-p} e^{\frac{\rho-1}{\rho} t}}{\rho^{-p} \Gamma(2-p)}
\end{aligned}
$$

Put $\delta_{1}(t)=\frac{t^{1-p}}{A_{1} \rho^{-p} \Gamma(2-p)}$ and $\delta_{2}(t)=\frac{A^{*} t^{1-p} e^{\frac{\rho-1}{\rho} t}}{\rho^{-} P(2-p)}$ and set $\bar{\delta}_{i}(t)=\max _{t \in[0,1]}\left\{\delta_{i}(t)\right\}, i=1,2$. Then we have

$$
\begin{aligned}
\left\|{ }^{C} D^{p, \rho} T x(t)\right\| \leq & \frac{\|\lambda\| t^{q-p}}{\rho^{q-p} \Gamma(q) \Gamma(2-p)} \\
& +\bar{\delta}_{1}(t)\left[\frac{\alpha_{2}\|\lambda\|}{\rho^{q-r} \Gamma(q-r+2)}\left(\eta^{q-r+1}-\zeta^{q-r+1}\right)\right. \\
& \left.+\frac{\alpha_{3}\|\lambda\|}{\rho^{q-r} \Gamma(q-r+1)} \sum_{i=1}^{m-2} \beta_{i} \gamma_{i}^{q-r}-\frac{\|\lambda\|}{\rho^{q-r} \Gamma(q-r+1)}\right] \\
& +\bar{\delta}_{2}(t) \\
= & M_{2} .
\end{aligned}
$$

Next, for $0<t_{1}<t_{2}<1$ and for all $x \in G$, we get

$$
\begin{aligned}
& \left|(T x)\left(t_{2}\right)-(T x)\left(t_{1}\right)\right| \\
& \leq \mid \frac{1}{\rho^{q} \Gamma(q)} \int_{0}^{t_{2}} e^{\frac{\rho-1}{\rho}\left(t_{2}-s\right)}\left(t_{2}-s\right)^{q-1} f\left(s, x(s), D^{p, \rho} x(s)\right) d s \\
& \quad-\frac{1}{\rho^{q} \Gamma(q)} \int_{0}^{t_{1}} e^{\frac{\rho-1}{\rho}\left(t_{1}-s\right)}\left(t_{1}-s\right)^{q-1} f\left(s, x(s), D^{p, \rho} x(s)\right) d s \mid \\
& \quad+\left|\mu_{1}\left(t_{2}\right)-\mu_{1}\left(t_{1}\right)\right|\left[\alpha_{2} \int_{\zeta}^{\eta}\left(\int_{0}^{s} \frac{\left|e^{\frac{\rho-1}{\rho}(s-u)}\right|(s-u)^{q-r-1}}{\rho^{q-r} \Gamma(q-r)}\left|f\left(u, x(u), D^{p, \rho} x(u)\right)\right| d u\right) d s\right. \\
& \quad+\alpha_{3} \sum_{i=1}^{m-2} \beta_{i} \int_{0}^{\gamma_{i}} \frac{\left|e^{\frac{\rho-1}{\rho}\left(\gamma_{i}-s\right)}\right|\left(\gamma_{i}-s\right)^{q-r-1}}{\rho^{q-r} \Gamma(q-r)}\left|f\left(s, x(s), D^{p, \rho} x(s)\right)\right| d s \\
& \left.\quad-\int_{0}^{1} \frac{\left|e^{\frac{\rho-1}{\rho}(1-s)}\right|(1-s)^{q-r-1}}{\rho^{q-r} \Gamma(q-r)}\left|f\left(s, x(s), D^{p, \rho} x(s)\right)\right| d s\right]+\left|\mu_{2}\left(t_{2}\right)-\mu_{2}\left(t_{1}\right)\right| \\
& =\frac{1}{\rho^{q} \Gamma(q)} \int_{0}^{t_{1}}\left|e^{\frac{\rho-1}{\rho}\left(t_{2}-s\right)}\right|\left(t_{2}-s\right)^{q-1}\left|f\left(s, x(s), D^{p, \rho} x(s)\right)\right| d s
\end{aligned}
$$




$$
\begin{aligned}
& +\frac{1}{\rho^{q} \Gamma(q)} \int_{t_{1}}^{t_{2}}\left|e^{\frac{\rho-1}{\rho}\left(t_{2}-s\right)}\right|\left(t_{2}-s\right)^{q-1}\left|f\left(s, x(s), D^{p, \rho} x(s)\right)\right| d s \\
& -\frac{1}{\rho^{q} \Gamma(q)} \int_{0}^{t_{1}}\left|e^{\frac{\rho-1}{\rho}\left(t_{1}-s\right)}\right|\left(t_{1}-s\right)^{q-1}\left|f\left(s, x(s), D^{p, \rho} x(s)\right)\right| d s \\
& +\left|\mu_{1}\left(t_{2}\right)-\mu_{1}\left(t_{1}\right)\right|\left[\alpha_{2} \int_{\zeta}^{\eta}\left(\int_{0}^{s} \frac{\left|e^{\frac{\rho-1}{\rho}(s-u)}\right|(s-u)^{q-r-1}}{\rho^{q-r} \Gamma(q-r)}\left|f\left(u, x(u), D^{p, \rho} x(u)\right)\right| d u\right) d s\right. \\
& +\alpha_{3} \sum_{i=1}^{m-2} \beta_{i} \int_{0}^{\gamma_{i}} \frac{\left|e^{\frac{\rho-1}{\rho}\left(\gamma_{i}-s\right)}\right|\left(\gamma_{i}-s\right)^{q-r-1}}{\rho^{q-r} \Gamma(q-r)}\left|f\left(s, x(s), D^{p, \rho} x(s)\right)\right| d s \\
& \left.-\int_{0}^{1} \frac{\left|e^{\frac{\rho-1}{\rho}(1-s)}\right|(1-s)^{q-r-1}}{\rho^{q-r} \Gamma(q-r)}\left|f\left(s, x(s), D^{p, \rho} x(s)\right)\right| d s\right]+\left|\mu_{2}\left(t_{2}\right)-\mu_{2}\left(t_{1}\right)\right| .
\end{aligned}
$$

Therefore, by the hypothesis of the theorem, we obtain

$$
\begin{aligned}
\left|(T x)\left(t_{2}\right)-(T x)\left(t_{1}\right)\right| \leq & \frac{\|\lambda\|}{\rho^{q} \Gamma(q+1)}\left\{\left[2\left(t_{2}-t_{1}\right)^{q}-\left(t_{2}^{q}-t_{1}^{q}\right)\right]\right. \\
& \left.+e^{\frac{\rho-1}{\rho}\left(t_{2}-t_{1}\right)}\left[\left(t_{2}^{q}-t_{1}^{q}\right)-\left(t_{2}-t_{1}\right)^{q}\right]\right\} \\
& +\frac{e^{\frac{\rho-1}{\rho} t_{2}}\left|t_{2}-t_{1}\right|}{\left|A_{1}\right|}\left[\frac{\alpha_{2}\|\lambda\|}{\rho^{q-r} \Gamma(q-r+2)}\left(\eta^{q-r+1}-\zeta^{q-r+1}\right)\right. \\
& \left.+\frac{\alpha_{3}\|\lambda\|}{\rho^{q-r} \Gamma(q-r+1)} \sum_{i=1}^{m-2} \beta_{i} \gamma_{i}^{q-r}-\frac{\|\lambda\|}{\rho^{q-r} \Gamma(q-r+1)}\right] \\
& +\left|A^{*}\right| e^{\frac{\rho-1}{\rho} t_{2}}\left|t_{2}-t_{1}\right| .
\end{aligned}
$$

In a similar way, we can get

$$
\begin{aligned}
\left|\left({ }^{C} D^{p, \rho} T x\right)\left(t_{2}\right)-\left({ }^{C} D^{p, \rho} T x\right)\left(t_{1}\right)\right| \leq & \frac{\|\lambda\|\left(t_{2}^{q-p}-t_{1}^{q-p}\right)}{\rho^{q-p} \Gamma(q) \Gamma(2-p)} \\
& +\left|\delta_{1}\left(t_{2}\right)-\delta_{1}\left(t_{1}\right)\right| \\
& {\left[\frac{\alpha_{2}\|\lambda\|}{\rho^{q-r} \Gamma(q-r+2)}\left(\eta^{q-r+1}-\zeta^{q-r+1}\right)\right.} \\
& +\frac{\alpha_{3}\|\lambda\|}{\rho^{q-r} \Gamma(q-r+1)} \sum_{i=1}^{m-2} \beta_{i} \gamma_{i}^{q-r} \\
& \left.-\frac{\|\lambda\|}{\rho^{q-r} \Gamma(q-r+1)}\right] \\
& +\left|\delta_{2}\left(t_{2}\right)-\delta_{2}\left(t_{1}\right)\right|
\end{aligned}
$$

where

$$
\begin{aligned}
& \left|\delta_{1}\left(t_{2}\right)-\delta_{1}\left(t_{1}\right)\right|=\frac{\left|t_{2}^{1-p}-t_{1}^{1-p}\right|}{\left|A_{1}\right| \rho^{-p} \Gamma(2-p)}, \\
& \left|\delta_{2}\left(t_{2}\right)-\delta_{2}\left(t_{1}\right)\right| \leq \frac{\left|A^{*}\right| e^{\frac{\rho-1}{\rho} t_{2}}\left|t_{2}^{1-p}-t_{1}^{1-p}\right|}{\rho^{-p} \Gamma(2-p)} .
\end{aligned}
$$


Then

$$
\begin{aligned}
\left|\left({ }^{C} D^{p, \rho} T x\right)\left(t_{2}\right)-\left({ }^{C} D^{p, \rho} T x\right)\left(t_{1}\right)\right| \leq & \frac{\|\lambda\|\left(t_{2}^{q-p}-t_{1}^{q-p}\right)}{\rho^{q-p} \Gamma(q) \Gamma(2-p)} \\
& +\frac{\left|t_{2}^{1-p}-t_{1}^{1-p}\right|}{\left|A_{1}\right| \rho^{-p} \Gamma(2-p)} \\
& {\left[\frac{\alpha_{2}\|\lambda\|}{\rho^{q-r} \Gamma(q-r+2)}\left(\eta^{q-r+1}-\zeta^{q-r+1}\right)\right.} \\
& +\frac{\alpha_{3}\|\lambda\|}{\rho^{q-r} \Gamma(q-r+1)} \sum_{i=1}^{m-2} \beta_{i} \gamma_{i}^{q-r} \\
& \left.-\frac{\|\lambda\|}{\rho^{q-r} \Gamma(q-r+1)}\right] \\
& +\frac{\left|A^{*}\right| e^{\frac{\rho-1}{\rho} t_{2}}\left|t_{2}^{1-p}-t_{1}^{1-p}\right|}{\rho^{-p} \Gamma(2-p)} .
\end{aligned}
$$

The functions $t^{q}, t, t^{q-p}, t^{1-p}$ are uniformly continuous on $[0,1]$ where $1 \leq q \leq 2$, $1-p>0, q-p>0$. Then, by the Arzela-Ascoli theorem, the sets $\{T(x): x \in G\}$ and $\left\{{ }^{C} D^{p, \rho} T(x): x \in G\right\}$ are relatively compact in $C[0,1]$. Therefore, $T(G)$ is a relatively compact set in $X$. Next, we consider the set

$$
K=\{x \in X: x=\varepsilon T x, 0<\varepsilon<1\} .
$$

Then $K$ is bounded. Indeed, let $x \in K$. So, $x=\varepsilon T x, 0<\varepsilon<1$. For any $t \in[0,1]$, it follows from $|x(t)|=\varepsilon|T x(t)|$ that

$$
\begin{aligned}
\|x\| \leq & \frac{\|\lambda\|}{\rho^{q} \Gamma(q+1)}+\bar{\mu}_{1}\left[\frac{\alpha_{2}\|\lambda\|}{\rho^{q-r} \Gamma(q-r+2)}\left|\eta^{q-r+1}-\zeta^{q-r+1}\right|\right. \\
& \left.+\frac{\alpha_{3}\|\lambda\|}{\rho^{q-r} \Gamma(q-r+1)} \sum_{i=1}^{m-2} \beta_{i} \gamma_{i}^{q-r}-\frac{\|\lambda\|}{\rho^{q-r} \Gamma(q-r+1)}\right]+\bar{\mu}_{2},
\end{aligned}
$$

which proves the boundedness of the set $K$. Thus, by Schaefer's fixed point theorem, the operator $T$ has at least one fixed point. Hence, the problem (1) has at least one solution on $[0,1]$, which completes the proof.

For our purpose, we write

$$
\begin{aligned}
& \bar{\theta}_{1}=\theta_{1}-\frac{1}{\rho^{q} \Gamma(q+1)}, \\
& \bar{\theta}_{2}=\theta_{2}-\frac{t^{q-p}}{\rho^{q-p} \Gamma(q) \Gamma(2-p)},
\end{aligned}
$$


where

$$
\begin{aligned}
\theta_{1}= & \frac{1}{\rho^{q} \Gamma(q+1)}\left(1+\mu_{1}(t)\left[\frac{\alpha_{2} \Gamma(q+1)}{\rho^{q-r} \Gamma(q-r+2)}\left(\eta^{q-r+1}-\zeta^{q-r+1}\right)\right.\right. \\
& \left.\left.+\frac{\alpha_{3} \Gamma(q+1)}{\rho^{q-r} \Gamma(q-r+1)} \sum_{i=1}^{m-2} \beta_{i} \gamma_{i}^{q-r}-\frac{\Gamma(q+1)}{\rho^{q-r} \Gamma(q-r+1)}\right]\right), \\
\theta_{2}= & \frac{t^{q-p}}{\rho^{q-p} \Gamma(q) \Gamma(2-p)}+\bar{\delta}_{1}(t)\left[\frac{\alpha_{2}}{\rho^{q-r} \Gamma(q-r+2)}\left(\eta^{q-r+1}-\zeta^{q-r+1}\right)\right. \\
& \left.+\frac{\alpha_{3}}{\rho^{q-r} \Gamma(q-r+1)} \sum_{i=1}^{m-2} \beta_{i} \gamma_{i}^{q-r}-\frac{1}{\rho^{q-r} \Gamma(q-r+1)}\right] .
\end{aligned}
$$

Next, we use Krasnoselskii's fixed point theorem to show the existence of solutions of the problem (1).

Theorem 16 Let $f:[0,1] \times \mathbb{R} \times \mathbb{R} \rightarrow \mathbb{R}$ be a continuous function such that the following conditions hold:

(H1) $|f(t, x, \tilde{x})-f(t, y, \tilde{y})|<L(|x-y|+|\tilde{x}+\tilde{y}|)$ for all $t \in[0,1], x, y, \tilde{x}, \tilde{y} \in \mathbb{R}, L>0$.

(H2) $\left|f\left(t, x(t),{ }^{C} D^{p, \rho} x(t)\right)\right| \leq c(t)$ for $t \in[0,1]$ and $c \in C\left([0,1], \mathbb{R}^{+}\right)$with $\|c\|=$ $\max _{t \in[0,1]}|c(t)|$.

(H3) $L \bar{\theta}<1$ where $\bar{\theta}=\max \left\{\bar{\theta}_{1}, \bar{\theta}_{2}\right\}$ and $\bar{\theta}_{1}, \bar{\theta}_{2}$ are given by (16) and (17).

Then there exists at least one solution for problem (1) on $[0,1]$.

Proof We define

$$
B_{r}=\{x \in X:\|x\| \leq r\}
$$

where $r \geq\|c\| \theta+v$ with

$$
\theta=\max \left\{\theta_{1}, \theta_{2}\right\} \quad \text { and } \quad v=\max \left\{\bar{\mu}_{2}, \bar{\delta}_{2}\right\}
$$

First, we split the operator $T$ given by (7) as $T=T_{1}+T_{2}$ on $B_{r}$ where

$$
\begin{aligned}
\left(T_{1} x\right)(t)= & \frac{1}{\rho^{q} \Gamma(q)} \int_{0}^{t} e^{\frac{\rho-1}{\rho}(t-s)}(t-s)^{q-1} f\left(s, x(s),{ }^{C} D^{p, \rho} x(s)\right) d s, \\
\left(T_{2} x\right)(t)= & \mu_{1}(t)\left[\alpha_{2} \int_{\zeta}^{\eta}\left(\int_{0}^{s} \frac{e^{\frac{\rho-1}{\rho}(s-u)}(s-u)^{q-r-1}}{\rho^{q-r} \Gamma(q-r)} f\left(u, x(u), D^{p, \rho} x(u)\right) d u\right) d s\right. \\
& +\alpha_{3} \sum_{i=1}^{m-2} \beta_{i} \int_{0}^{\gamma_{i}} \frac{e^{\frac{\rho-1}{\rho}\left(\gamma_{i}-s\right)}\left(\gamma_{i}-s\right)^{q-r-1}}{\rho^{q-r} \Gamma(q-r)} f\left(s, x(s), D^{p, \rho} x(s)\right) d s \\
& \left.-\int_{0}^{1} \frac{e^{\frac{\rho-1}{\rho}(1-s)}(1-s)^{q-r-1}}{\rho^{q-r} \Gamma(q-r)} f\left(s, x(s), D^{p, \rho} x(s)\right) d s\right]+\mu_{2}(t) .
\end{aligned}
$$


For $\widehat{x}, \widehat{y} \in B_{r}$ and using (18), we can get

$$
\begin{aligned}
\left\|T_{1}(\widehat{x})-T_{2}(\widehat{x})\right\| \leq & \frac{\|c\|}{\rho^{q} \Gamma(q+1)}+\bar{\mu}_{1}\left[\frac{\alpha_{2}\|c\|}{\rho^{q-r} \Gamma(q-r+2)}\left(\eta^{q-r+1}-\zeta^{q-r+1}\right)\right. \\
& \left.+\frac{\alpha_{3}\|c\|}{\rho^{q-r} \Gamma(q-r+1)} \sum_{i=1}^{m-2} \beta_{i} \gamma_{i}^{q-r}-\frac{\|c\|}{\rho^{q-r} \Gamma(q-r+1)}\right] \\
& +\bar{\mu}_{2}
\end{aligned}
$$

and

$$
\begin{aligned}
\left\|\left({ }^{C} D^{p, \rho} T_{1}\right)(\widehat{x})-\left({ }^{C} D^{p, \rho} T_{2}\right)(\widehat{x})\right\| \leq & \frac{\|c\| t^{q-p}}{\rho^{q-p} \Gamma(q) \Gamma(2-p)} \\
& +\bar{\delta}_{1}(t)\left[\frac{\alpha_{2}\|c\|}{\rho^{q-r} \Gamma(q-r+2)}\left(\eta^{q-r+1}-\zeta^{q-r+1}\right)\right. \\
& +\frac{\alpha_{3}\|c\|}{\rho^{q-r} \Gamma(q-r+1)} \sum_{i=1}^{m-2} \beta_{i} \gamma_{i}^{q-r} \\
& \left.-\frac{\|c\|}{\rho^{q-r} \Gamma(q-r+1)}\right]+\bar{\delta}_{2}(t) .
\end{aligned}
$$

Then, by (11), we obtain

$$
\left\|T_{1}(\widehat{x})-T_{2}(\widehat{x})\right\| \leq\|c\| \theta+v \leq r
$$

and

$$
\left\|\left({ }^{C} D^{p, \rho} T_{1}\right)(\widehat{x})-\left({ }^{C} D^{p, \rho} T_{2}\right)(\widehat{x})\right\| \leq\|c\| \delta+v \leq r,
$$

which shows that $T_{1}(\widehat{x})-T_{2}(\widehat{x}) \in B_{r}$. Next, we show that $T_{2}$ is a contraction. Let $x, y \in \mathbb{R}$, $t \in[0,1]$. Then, by using $(\mathrm{H} 1)$, we have

$$
\begin{aligned}
& \left\|T_{2}(x)-T_{2}(y)\right\| \\
& =\sup _{t \in[0,1]}\left|T_{2}(x)-T_{2}(y)\right| \\
& =\sup _{t \in[0,1]} \mid\left(\mu _ { 1 } ( t ) \left[\alpha_{2} \int_{\zeta}^{\eta}\left(\int_{0}^{s} \frac{e^{\frac{\rho-1}{\rho}(s-u)}(s-u)^{q-r-1}}{\rho^{q-r} \Gamma(q-r)} f\left(u, x(u), D^{p, \rho} x(u)\right) d u\right) d s\right.\right. \\
& \quad+\alpha_{3} \sum_{i=1}^{m-2} \beta_{i} \int_{0}^{\gamma_{i}} \frac{e^{\frac{\rho-1}{\rho}\left(\gamma_{i}-s\right)}\left(\gamma_{i}-s\right)^{q-r-1}}{\rho^{q-r} \Gamma(q-r)} f\left(s, x(s), D^{p, \rho} x(s)\right) d s \\
& \left.\left.\quad-\int_{0}^{1} \frac{e^{\frac{\rho-1}{\rho}(1-s)}(1-s)^{q-r-1}}{\rho^{q-r} \Gamma(q-r)} f\left(s, x(s), D^{p, \rho} x(s)\right) d s\right]+\mu_{2}(t)\right) \\
& \quad-\left(\mu _ { 1 } ( t ) \left[\alpha_{2} \int_{\zeta}^{\eta}\left(\int_{0}^{s} \frac{e^{\frac{\rho-1}{\rho}(s-u)}(s-u)^{q-r-1}}{\rho^{q-r} \Gamma(q-r)} f\left(u, y(u), D^{p, \rho} y(u)\right) d u\right) d s\right.\right.
\end{aligned}
$$




$$
\begin{aligned}
& +\alpha_{3} \sum_{i=1}^{m-2} \beta_{i} \int_{0}^{\gamma_{i}} \frac{e^{\frac{\rho-1}{\rho}\left(\gamma_{i}-s\right)}\left(\gamma_{i}-s\right)^{q-r-1}}{\rho^{q-r} \Gamma(q-r)} f\left(s, y(s), D^{p, \rho} y(s)\right) d s \\
& \left.\left.-\int_{0}^{1} \frac{e^{\frac{\rho-1}{\rho}(1-s)}(1-s)^{q-r-1}}{\rho^{q-r} \Gamma(q-r)} f\left(s, y(s), D^{p, \rho} y(s)\right) d s\right]+\mu_{2}(t)\right) \mid \\
\leq & \sup _{t \in[0,1]} L\left[|x(t)-y(t)|+\left|{ }^{C} D^{p, \rho} x(t)-{ }^{C} D^{p, \rho} y(t)\right|\right] \\
& \left\{| \mu _ { 1 } ( t ) | \left[\alpha_{2} \int_{\zeta}^{\eta}\left(\int_{0}^{s} \frac{(s-u)^{q-r-1}}{\rho^{q-r} \Gamma(q-r)} d u\right) d s\right.\right. \\
& \left.\left.+\alpha_{3} \sum_{i=1}^{m-2} \beta_{i} \int_{0}^{\gamma_{i}} \frac{\left(\gamma_{i}-s\right)^{q-r-1}}{\rho^{q-r} \Gamma(q-r)} d s-\int_{0}^{1} \frac{(1-s)^{q-r-1}}{\rho^{q-r} \Gamma(q-r)} d s\right]\right\} \\
\leq & L\|x-y\| \sup _{t \in[0,1]}\left\{| \mu _ { 1 } ( t ) | \left[\alpha_{2} \int_{\zeta}^{\eta}\left(\int_{0}^{s} \frac{(s-u)^{q-r-1}}{\rho^{q-r} \Gamma(q-r)} d u\right) d s\right.\right. \\
& \left.\left.+\alpha_{3} \sum_{i=1}^{m-2} \beta_{i} \int_{0}^{\gamma_{i}} \frac{\left(\gamma_{i}-s\right)^{q-r-1}}{\rho^{q-r} \Gamma(q-r)} d s-\int_{0}^{1} \frac{(1-s)^{q-r-1}}{\rho^{q-r} \Gamma(q-r)} d s\right]\right\} \\
\leq & L \bar{\theta}_{1}\|x-y\| \leq L \bar{\theta}\|x-y\|,
\end{aligned}
$$

where

$$
\bar{\theta}_{1}=\theta_{1}-\frac{1}{\rho^{q} \Gamma(q+1)} .
$$

Similarly,

$$
\left\|\left({ }^{C} D^{p, \rho} T_{2}\right)(x)-\left({ }^{C} D^{p, \rho} T_{2}\right)(y)\right\| \leq L \bar{\theta}_{2}\|x-y\| \leq L \bar{\theta}\|x-y\|,
$$

where

$$
\bar{\theta}_{2}=\theta_{2}-\frac{t^{q-p}}{\rho^{q-p} \Gamma(q) \Gamma(2-p)} .
$$

Therefore, by (H3), the operator $T_{2}$ is a contraction. It remains to show that $T_{1}$ is continuous and compact. We have

$$
\left(T_{1} x\right)(t)=\frac{1}{\rho^{q} \Gamma(q)} \int_{0}^{t} e^{\frac{\rho-1}{\rho}(t-s)}(t-s)^{q-1} f\left(s, x(s),{ }^{C} D^{p, \rho} x(s)\right) d s .
$$

Then, by the continuity of $f$, the operator $T_{1}$ is continuous. Also,

$$
\begin{aligned}
\left\|T_{1}(x)\right\| & =\frac{1}{\rho^{q} \Gamma(q)} \sup _{t \in[0,1]} \int_{0}^{t}\left|e^{\frac{\rho-1}{\rho}(t-s)}\right|(t-s)^{q-1}\left|f\left(s, x(s),{ }^{C} D^{p, \rho} x(s)\right)\right| d s \\
& \leq \frac{\|c\|}{\rho^{q} \Gamma(q+1)} .
\end{aligned}
$$


Further,

$$
\begin{aligned}
\left\|\left({ }^{C} D^{p, \rho} T_{1}\right)(x)\right\| & =\sup _{t \in[0,1]}\left(\frac{\|c\| t^{q-p}}{\rho^{q-p} \Gamma(q) \Gamma(2-p)}\right) \\
& \leq \frac{\|c\|}{\rho^{q-p} \Gamma(q) \Gamma(2-p)} .
\end{aligned}
$$

Now, for $t_{1}<t_{2}$ and $t_{1}, t_{2} \in(0,1]$ with $\sup _{(t, x, y) \in[0,1] \times B_{r} \times B_{r}}|f(t, x, y)|=w$, we have

$$
\begin{aligned}
\left|\left(T_{1} x\right)\left(t_{2}\right)-\left(T_{1} x\right)\left(t_{1}\right)\right| \leq & \frac{w}{\rho^{q} \Gamma(q+1)}\left\{\left[2\left(t_{2}-t_{1}\right)^{q}-\left(t_{2}^{q}-t_{1}^{q}\right)\right]\right. \\
& \left.+e^{\frac{\rho-1}{\rho}\left(t_{2}-t_{1}\right)}\left[\left(t_{2}^{q}-t_{1}^{q}\right)-\left(t_{2}-t_{1}\right)^{q}\right]\right\}
\end{aligned}
$$

and

$$
\left|\left({ }^{C} D^{p, \rho} T_{1} x\right)\left(t_{2}\right)-\left({ }^{C} D^{p, \rho} T_{1} x\right)\left(t_{1}\right)\right| \leq \frac{w\left|t_{2}^{q-p}-t_{1}^{q-p}\right|}{\rho^{q-p} \Gamma(q) \Gamma(2-p)} .
$$

Therefore, as $\left(t_{2}-t_{1}\right) \rightarrow 0$, the right-hand sides of (25) and (26) tend to zero independent of $x$. Thus, $T_{1}$ is equicontinuous and so it is relatively compact on $B_{r}$ according to the Arzela-Ascoli theorem. Then the operator $T_{1}$ is compact. By using Krasnoselskii's fixed point theorem, there exists at least one solution of $(7)$ on $[0,1]$, and the proof is complete.

The following example shows the applicability of Theorem 15 .

Example Consider the problem

$$
\begin{aligned}
& { }^{C} D^{q, \rho} x(t)=(t+1-|x(t)|) \ln (t+1-|x(t)|)+\frac{\left|D^{p, \rho} x(t)\right|}{1+\left|D^{p, \rho} x(t)\right|}, \quad t \in[0,1], \\
& x(0)=\alpha_{1}, \\
& { }^{C} D^{r, \rho} x(1)=\alpha_{2} \int_{\zeta}^{\eta}{ }^{C} D^{r, \rho} x(s) d s+\alpha_{3} \sum_{i=1}^{m-2} \beta_{i}{ }^{C} D^{r, \rho} x\left(\gamma_{i}\right),
\end{aligned}
$$

where $1<q \leq 2, p \in(0,1)$ and $r \in(0,1)$. Here,

$$
f\left(t, x, D^{p, \rho} x(t)\right)=(t+1-|x(t)|) \ln (t+1-|x(t)|)+\frac{\left|D^{p, \rho} x(t)\right|}{1+\left|D^{p, \rho} x(t)\right|}
$$

and then

$$
\left|f\left(t, x, D^{p, \rho} x(t)\right)\right|<(t+1) \ln (t+1)+1 .
$$

If we take $F(u)=e^{u^{2}}-1$, then $F$ is an $N$-function satisfying

$$
\int_{0}^{1} F(|f(u(x))|) d x<\infty
$$

from which it follows that $F$ belongs to the Orlicz space $L_{F}[0,1]$. Observe that

$$
\left|f\left(t, x, D^{p, \rho} x(t)\right)\right|<\lambda(t)
$$


where $\lambda(t)=(t+1) \ln (t+1)+1$. Therefore, Theorem 15 applies and there exists a solution for a problem (1) on $[0,1]$.

\author{
Acknowledgements \\ Not applicable. \\ Funding \\ Not applicable.

\section{Availability of data and materials} \\ Not applicable.
}

Ethics approval and consent to participate

Not applicable.

\title{
Competing interests
}

The authors declare that they have no competing interests.

\section{Consent for publication}

Not applicable.

\section{Authors' contributions}

All authors read and approved the final manuscript.

\section{Publisher's Note}

Springer Nature remains neutral with regard to jurisdictional claims in published maps and institutional affiliations.

Received: 13 November 2018 Accepted: 22 February 2019 Published online: 06 March 2019

\section{References}

1. Machado, J.T., Kiryakova, V., Mainardi, F.: Recent history of fractional calculus. Commun. Nonlinear Sci. Numer. Simul. 16, 1140-1153 (2011)

2. Zhao, K., Wang, K.: Existence of solutions for the delayed nonlinear fractional functional differential equations with three-point integral boundary value conditions. Adv. Differ. Equ. 2016, 284 (2016)

3. Li, Y., Qi, A.: Existence of positive solutions for multi-point boundary value problems of Caputo differential equation. Int. J. Dyn. Syst. Differ. Equ. 7(2), 169-183 (2017)

4. Cui, M., Zhu, Y., Pang, H.: Existence and uniqueness results for a coupled fractional order systems with the multi-strip and multi-point mixed boundary conditions. Adv. Differ. Equ. 2017, 224 (2017)

5. Wang, Y., Liang, S., Wang, Q.: Existence results for fractional differential equations with integral and multi-point boundary conditions. Bound. Value Probl. 2018, 4 (2018)

6. Agarwal, R.P., Alsaedi, A., Alsharif, A., Ahmad, B.: On nonlinear fractional-order boundary value problems with nonlocal multi-point conditions involving Liouville-Caputo derivatives. Differ. Equ. Appl. 9(2), 147-160 (2017)

7. Caputo, M., Fabrizio, M.: A new definition of fractional derivative without singular kernel. Prog. Fract. Differ. Appl. 1(2), 73-85 (2015)

8. Atangana, A., Baleanu, D.: New fractional derivatives with nonlocal and non-singular kernel: theory and application to heat transfer model. Therm. Sci. 20(2), 763-769 (2016)

9. Jarad, F., Abdeljawad, T., Alzabut, J.: Generalized fractional derivatives generated by a class of local proportional derivatives. Eur. Phys. J. Spec. Top. 226, 3457-3471 (2017)

10. Khalil, R., Al Horani, M., Yousef, A., Sababheh, M.: A new definition of fractional derivative. J. Comput. Appl. Math. 264, 65-70 (2014)

11. Anderson, D.R., Ulness, D.J.: Newly defined conformable derivatives. Adv. Dyn. Syst. Appl. 10, 109-137 (2015)

12. Anderson, D.R.: Second-order self-adjoint differential equations using a proportional derivative controller. Commun. Appl. Nonlinear Anal. 24, 17-48 (2017)

13. Jarad, F., Abdeljawad, T., Baleanu, D.: On the generalized fractional derivatives and their Caputo modification. J. Nonlinear Sci. Appl. 10, 2607-2619 (2017)

14. Abdeljawad, T.: On conformable fractional calculus. J. Comput. Appl. Math. 279, 57-66 (2015)

15. Abdeljawad, T., Jarad, F., Alzabut, J.: Fractional proportional differences with memory. Eur. Phys. J. Spec. Top. 266(1618), 3333-3354 (2017)

16. Jarad, F., Abdeljawad, T., Hammouch, Z:: On a class of ordinary differential equations in the frame of Atangana-Baleanu fractional derivative. Chaos Solitons Fractals 117, 16-20 (2018)

17. Abdeljawad, T., Al-Mdallal, Q.M., Hajji, M.A.: Arbitrary order fractional difference operators with discrete exponential kernels and applications. Discrete Dyn. Nat. Soc. 2017, Article ID 4149320 (2017)

18. Abdeljawad, T.: Fractional operators with exponential kernels and a Lyapunov type inequality. Adv. Differ. Equ. 2017 $313(2017)$

19. Ahmad, B., Ntouyas, S.K., Alsaedi, A., Shammakh, W., Agarwal, R.P.: Existence theory for fractional differential equations with non-separated type nonlocal multi-point and multi-strip boundary conditions. Adv. Differ. Equ. 2018, 89 (2018)

20. Alsaedi, A., Alsulami, M., Agarwal, R.P., Ahmad, B.: Some new nonlinear second-order boundary value problems on an arbitrary domain. Adv. Differ. Equ. 2018, 227 (2018) 
21. Birnbaum, Z.W., Orlicz, W.: Über die Verallgemeinerung des Begriffes der zueinander Konjugierten Potenzen. Stud. Math. 3, 1-67 (1931)

22. Ayazoglu, R., Avci, M., Chung, N.T.: Existence of solutions for nonlocal problems in Orlicz-Sobolev spaces via monotone method. Electron. J. Math. Anal. Appl. 4(1), 63-73 (2016)

23. Mihailescu, M., Pepovs, D.: Multiple solutions for a nonlinear and non-homogenous problem in Orlicz-Sobolev spaces (2016). arXiv:1603.05042v1

24. Adams, R.A.: Orlicz and Orlicz-Sobolev spaces. Pure Appl. Math. 65, 227-259 (1975)

25. Kilbas, A.A., Srivastava, H.M., Trujillo, J.J.: Thoery and Applications of Fractional Differential Equations. North-Holand Mathematics Studies, vol. 24. Elsevier, Amsterdam (2006)

26. Podlubny, L.: Fractional Differential Equations. Academic Press, San Diego (1999)

27. Samko, S.G., Kilbas, A.A., Marichev, O.I.: Fractional Integrals and Derivatives: Theory and Applications, Switzerland (1993)

28. Smart, D.R.: Fixed Point Theorems. Cambridge University Press, Cambridge (1980)

Submit your manuscript to a SpringerOpen ${ }^{\circ}$ journal and benefit from:

- Convenient online submission

- Rigorous peer review

Open access: articles freely available online

- High visibility within the field

- Retaining the copyright to your article

Submit your next manuscript at $\boldsymbol{\nabla}$ springeropen.com 